\title{
DIVERSIDAD GENÉTICA EN MÉXICO DE VARIEDADES NATIVAS DE CHILE 'POBLANO’ MEDIANTE MICROSATÉLITES
}

\author{
GENETIC DIVERSITY IN MEXICO OF 'POBLANO’ PEPPER LANDRACES BY MICROSATELLITES
}

\author{
Aremi R. Contreras Toledo ${ }^{1}$, Higinio López Sánchez ${ }^{2}$, Amalio Santacruz Varela ${ }^{1 \star}$, Ernestina Valadez \\ Moctezuma $^{3}$, Víctor H. Aguilar Rincón ${ }^{1}$, Tarsicio Corona Torres ${ }^{1}$ y Pedro Antonio López ${ }^{2}$
}

\begin{abstract}
${ }^{1}$ Colegio de Postgraduados-Campus Montecillo. Carr. México-Texcoco, km. 36.5. 56230, Montecillo, Texcoco, Estado de México, México. ${ }^{2}$ Colegio de PostgraduadosCampus Puebla. Carr. México- Puebla, km. 125.5. Santiago Momoxpan, Puebla, México. ${ }^{3}$ Universidad Autónoma Chapingo. Carr. México-Texcoco, km 38.5. 56230, Chapingo, Estado de México, México.
\end{abstract}

*Autor para correspondencia (asvarela@colpos.mx)

\section{RESUMEN}

La domesticación del chile (Capsicum annuum L.) se efectuó en México, gracias a lo cual se encuentra una gran riqueza de variedades en el país. En el centro del país se encuentra distribuido el chile 'Poblano', que no es consumido por su contenido de capsaicina, como la mayoría de las especies del género, sino como ingrediente principal de platillos tradicionales. Este estudio se hizo para describir los diferentes grupos genéticos que forman las variedades, determinar sus posibles patrones de distribución de diversidad, analizar la estructura genética de las poblaciones de chile 'Poblano' y su relación con otros tipos de chile. Se evaluaron 55 poblaciones de chile 'Poblano', 2 de 'Loco', 2 de 'Miahuateco' y 3 de 'Ancho', colectadas en el Valle de Puebla, Tehuacán, Puebla y Rancho Grande, Zacatecas, más un híbrido comercial como testigo. Se utilizaron 19 loci de microsatélites (SSR) y se calcularon los parámetros de diversidad genética, proporción de loci polimórficos, índice de heterocigosidad y estadísticos de F de Wright; además, se hicieron análisis de componentes principales y de conglomerados. Se detectaron 105 alelos en total, con un promedio de 5.53 alelos por locus y 80 \% de loci polimórficos. Las variedades locales destacaron por ser las de mayor polimorfismo y heterocigosidad. El estadístico $F_{\mathrm{ST}}$ de diferenciación genética fue de 0.108 , que indica que $89.2 \%$ de la variación se encuentra dentro de las poblaciones. Hubo mayor diferenciación en los tipos 'Poblano', 'Ancho' y 'Loco'. Las diferentes poblaciones formaron grupos definidos con cierta dispersión dentro del tipo 'Poblano'. Se detectó alta diferenciación entre las variedades provenientes del Valle de Puebla, Tehuacán y Zacatecas, aparte del híbrido comercial. La complejidad genética fue mayor en las variedades locales, que no presentaron un patrón de distribución.

Palabras clave: Capsicum annuum, SSR, polimorfismo, diferenciación genética.

\section{SUMMARY}

Domestication of chili pepper (Capsicum annuum L.) took place in México, producing large diversity of varieties in the country. 'Poblano' pepper is distributed over central México, and it is not consumed by virtue of its capsaicin content, as most of the species of the genus, but by its use as the main ingredient of traditional dishes. In order to describe the different genetic groups composed by varieties, to determine their possible patterns of distribution of diversity, to analyze the genetic structure of 'Poblano' pepper populations and their relationships with other pepper types, this study was carried out. The research considered 63 pepper populations: 55 of 'Poblano' pepper, 2 of 'Loco' pepper, 2 of 'Miahuateco' pepper and 3 of 'Ancho' pepper, collected from the Puebla Valley, Tehuacán, Puebla and Rancho Grande, Zacatecas, and the commercial hybrid 'Doroteo" as reference. Nineteen microsatellite (SSR) loci were analyzed for calculating the following genetic diversity parameters: proportion of polymorphic loci, heterozygosity and Wright's F-Statistics; in addition, principal components and cluster analyses were performed. A total of 105 alleles were detected with an average of 5.53 alleles per locus and $80 \%$ of the loci were found to be polymorphic, with local landraces showing higher polymorphism and heterozygosity. The genetic differentiation $\mathrm{F}_{\mathrm{ST}}$ was 0.108 , which indicates that $89.2 \%$ of the variation is within populations, with a major differentiation into the 'Poblano', 'Ancho' and 'Loco' pepper types. Populations formed well defined groups, but some dispersion was observed within the 'Poblano' group. A high differentiation among varieties from the Puebla Valley, Tehuacán and Zacatecas was detected, aside the commercial hybrid. The genetic complexity was superior within the pepper landraces, which did not show a defined pattern of distribution.

Index words: Capsicum annuum, SSR, polymorphism, genetic differentiation.

\section{INTRODUCCIÓN}

Capsisum annuum L. es conocida como chile en México, y en el mundo es la especie más importante del género Capsicum (Solanacae) por la superficie cultivada y por los beneficios económicos que ofrece, además de su alta demanda por su valor nutricional. Debido a lo anterior, durante las últimas tres décadas se ha incrementando la producción de chile a nivel mundial, pues en 1990 se cosecharon 12.8 millones de toneladas de fruto verde y seco, que pasaron a 23.1 millones en el año 2000 y a 31.2 millones en 2009 (FAOSTAT, 2009), principalmente para su uso como especia, hortaliza y para su industrialización en la obtención de colorantes (Djian-Caporalino et al., 2006). A nivel mundial el chile se cultiva en aproximadamente 3.7 
millones de hectáreas anuales, con China, México y Turquía como los principales países productores (FAOSTAT, 2009). Estudios arqueológicos han revelado que el chile es uno de los cultivos ancestrales de Centro, Sudamérica y México, donde el proceso de domesticación comenzó entre 7000 y 5000 años a.C., probablemente en el Valle de Tehuacán, Puebla (Pickersgill, 1991).

A partir de la domesticación de chile se ha generado en México una gran diversidad dentro de esta especie, debido a la selección que efectúan los agricultores para diferentes usos. De la variabilidad de tipos de C. annuum L., el chile 'Poblano' es uno de los más importantes y representativos del Estado de Puebla, con una presencia histórica y cultural indiscutible (Rodríguez et al., 2007). En el Estado de Puebla, la superficie cultivada con chile 'Poblano' es de 600 ha, con una producción de 4800 t (SDR Puebla, 2007), superficie en la que existe una gran diversidad de variedades criollas, originada por la siembra ininterrumpida por los agricultores de sus propias semillas, lo que ha generado una vasta complejidad en tipos, formas y colores (Rodríguez et al., 2007).

Esta diversidad genética está en riesgo de perderse debido a una problemática asociada con aspectos como: falta de semilla mejorada a partir de germoplasma local y con criterios de los propios productores; presencia de enfermedades, como la "secadera del chile" (Phytophthora capsici, Fusarium oxysporum); e incidencia de factores abióticos adversos como heladas tempranas. Esos factores han afectado el rendimiento de fruto, situación que podría llegar a provocar el abandono del cultivo (Rodríguez et al., 2007). A pesar de la importancia de la diversidad genética y del riesgo de perder las variedades nativas de chile 'Poblano', no hay estudios que permitan conocer su nivel actual y con ello diseñar programas de conservación y aprovechamiento de estos recursos.

El objetivo de la presente investigación fue describir la diversidad genética de las variedades locales de chile 'Poblano' de la Sierra Nevada de Puebla, mediante el uso de microsatélites (SSR), lo cual permitirá diferenciar grupos genéticos de variedades, determinar posibles patrones de distribución de diversidad, analizar la estructura genética de estas poblaciones, y estudiar su relación con otros tipos de chile de la región y de otras partes del país.

\section{MATERIALES Y MÉTODOS}

\section{Material vegetal}

Se colectaron 55 poblaciones de variedades locales de chile 'Poblano', dos de chile 'Loco', dos de chile 'Miahuateco' y tres de chile 'Ancho', procedentes del Valle de Puebla, Tehuacán, Pue. y Rancho Grande, Zacatecas, además del híbrido comercial 'Doroteo' (Ahern International de México, S.A. de C.V.) que es del tipo 'Ancho' (Cuadro 1). Los genotipos de chile 'Ancho' se incluyeron con el fin de diferenciarlos genéticamente del chile 'Poblano', pues con frecuencia se les confunde y llegan a comercializarse con denominaciones intercambiadas.

Cuadro 1. Localidades de colecta por tipo de chile (Capsicum annuum L.), de los materiales incluidos en el estudio de diversidad genética.

\begin{tabular}{|c|c|c|c|c|c|}
\hline \multirow{2}{*}{ Localidad } & \multicolumn{5}{|c|}{ Tipos de chile ${ }^{\dagger}$} \\
\hline & 'Poblano' & 'Ancho' & 'Loco' & 'Miahuateco' & Híbrido \\
\hline Cháhuac & CH1-P, CH2-P, CH3-P, CH4-P & & & & \\
\hline Domingo Arenas & DA1-P & & & & \\
\hline San Felipe Teotlatzingo & FT1-P, FT2-P, FT3-P, FT5-P, FT6-P & & FT4-L & & \\
\hline San Lorenzo Chiautzingo & LC1-P, LC2-P, LC3-P, LC4-P, LC5-P, LC6-P & & & & \\
\hline San Lucas El Grande & LG1-P, LG2-P, LG3-P, LG4-P, LG5-P & & & & \\
\hline San Mateo Capultitlán & MC1-P, MC2-P, MC3-P, MC4-P, MC5-P, MC6-P & MC8-A & MC7-L & & \\
\hline Juárez Coronaco & JC1-P, JC2-P, JC3-P, JC4-P, JC5-P & & & & \\
\hline San Antonio Chautla & AC1-P & & & & \\
\hline San Matías Tlalancaleca & MT1-P, MT2-P, MT3-P, MT4-P, MT5-P, MT6-P, MT7-P & & & & \\
\hline San Miguel Tianguistengo & TI1-P, TI2-P & & & & \\
\hline San Rafael Tlanalapan & RT1-P, RT2-P, RT3-P, RT4-P, RT5-P, RT6-P, RT7-P, RT8-P, RT9-P & & & & \\
\hline Santa María Moyotzingo & MO1-P, MO2-P, MO3-P, MO4-P & & & & \\
\hline Santiago Miahuatlán, Tehuacán & & & & MI1-M, MI2-M & \\
\hline Rancho Grande, Zacatecas & & RG1-A, RG2-A & & & \\
\hline Ahern Int. S.A. de C.V. & & & & & 'Doroteo' \\
\hline Total & 55 & 3 & 2 & 2 & 1 \\
\hline
\end{tabular}

${ }^{\dagger}$ Las dos primeras letras de las poblaciones identifican la localidad o procedencia, el número, el orden de la población y la letra en seguida del guión el tipo de chile. 


\section{Extracción de ADN y amplificación de microsatélites}

Se hizo la extracción de ADN total a partir de tejido de hoja de 10 plántulas por población con el kit comercial Wizard Genomic DNA Purification ${ }^{\circledR}$ (Promega). Se amplificaron 19 loci de microsatélites (Lee et al., 2004) por PCR múltiple, y los iniciadores se marcaron con las etiquetas fluorescentes 6-FAM o HEX (Applied Biosystems) en el extremo 5, para su detección en secuenciador de fragmentos. Los iniciadores no asignados a un grupo fueron amplificados de forma individual (Cuadro 2). La amplificación en PCR múltiple se llevó a cabo en mezclas de reacción de $25 \mu \mathrm{L}$ que contenían $0.2 \mathrm{mM}$ de nucleótidos, $4 \mathrm{mM}$ de $\mathrm{MgCl}_{2}, 1.6 \mathrm{X}$ de amortiguador, $40 \mathrm{ng}$ de ADN, 1 unidad de Taq ADN polimerasa y 15 pmol de cada iniciador. La amplificación se hizo con una desnaturalización inicial de $4 \min$ a $94^{\circ} \mathrm{C}, 35$ ciclos de 1 min a $94{ }^{\circ} \mathrm{C}, 1 \min$ a $65^{\circ} \mathrm{C}, 2 \min$ a $72{ }^{\circ} \mathrm{C}$ y una extensión final de $12 \mathrm{~min}$ a $72{ }^{\circ} \mathrm{C}$. Los productos de PCR se evaluaron por electroforesis capilar en un secuenciador Genetic Analyzer 3130® (Applied Biosystems) y analizados con el programa GeneMapper® V. 4.0 (Applied Biosystems, 2005).

\section{Manejo de datos y análisis estadístico}

Se registraron los diferentes alelos detectados en cada individuo para cada locus, con la finalidad de obtener las frecuencias alélicas poblacionales; adicionalmente, se contabilizó el número de alelos por locus, proporción de loci polimórficos, índice de heterocigosidad esperada y los estadísticos de $\mathrm{F}$ de Wright $\mathrm{F}_{\mathrm{IS}}$ (medida de la desviación de heterocigosidad dentro de accesiones con respecto a la esperada con base en las frecuencias alélicas de la accesión bajo apareamiento aleatorio), $\mathrm{F}_{\mathrm{ST}}$ (reducción en heterocigosidad esperada bajo apareamiento aleatorio en un nivel jerárquico en relación con otro nivel superior, atribuible a la diferenciación de accesiones en los grupos genéticos) y $\mathrm{F}_{\text {IT }}$ (coeficiente global de endogamia), con el programa POPGENE (Yeh et al., 1999). Se hizo además un análisis de componentes principales con base en la matriz de correlaciones generada a partir de las frecuencias alélicas con el paquete SAS V.9.0. (SAS Institute, 2002). De manera complementaria, se efectúo un análisis de conglomerados con el programa NTSYSpc ${ }^{\circledR}$ V.2.1 (Rohlf, 2002) mediante la distancia genética de Rogers modificada por Wright (1978) y el método de agrupamiento UPGMA (unweighted pair group method with arithmetic mean).

\section{RESULTADOS Y DISCUSIÓN}

\section{Variabilidad genética en chile 'Poblano'}

Con excepción del locus HpmsAT2-14, todos los loci fueron polimórficos, con valores del contenido de información de polimorfismo (PIC, por sus siglas en inglés) entre 0 y 0.66 (Cuadro 2). Se detectaron 105 alelos en los 19 loci estudiados en todas las poblaciones. En el presente estudio se utilizaron 11 loci de microsatélites en común con Kwon et al. (2005). Estos autores estudiaron la variación molecular de híbridos de chile, y encontraron que los intervalos de tamaño de fragmento eran muy similares, y la mayoría de ellos registró un menor número de alelos que los obtenidos en el presente estudio, posiblemente debido a la estrecha base genética de las variedades híbridas estudiadas por estos autores. No obstante, los valores de PIC fueron más altos, debido a que en estos materiales existe una mayor probabilidad de presentar dos alelos diferentes por locus, dada su constitución genética altamente heterocigótica.

Se detectaron 32 alelos exclusivos de poblaciones de chile, los cuales ocurrieron en baja frecuencia y representaron 30 $\%$ de los alelos totales encontrados. La proporción de loci polimórficos fue de $94.7 \%$ en los tipos 'Poblano', 'Ancho' y 'Miahuateco', $73.7 \%$ en 'Loco' y $42.1 \%$ en el hibrido 'Doroteo', lo que indica una mayor diversidad de alelos en los primeros tres tipos de chile. El promedio de alelos por locus fue de 5.53 con $80 \%$ de loci polimórficos (Cuadro 3). En su estudio Hanáček et al. (2009) analizaron 41 accesiones de chile 'Rojo', con algunos de los iniciadores utilizados también en el presente estudio, aunque fueron más escasos en chile 'Rojo', ya que el número de alelos por locus fue de 3.5, con $62.5 \%$ de loci polimórficos. Kwon et al. (2005) encontraron un promedio de 3.3 alelos por locus en variedades mejoradas de chile. Hernández et al. (2006) obtuvieron resultados contrastantes en poblaciones domesticadas de los chiles 'Serrano', 'Jalapeño' y 'Morrón', analizadas con isoenzimas y RAPDs, marcadores que indicaron polimorfismos de 84.6 y $34.7 \%$, respectivamente. Por su parte, Tam et al. (2005) encontraron una heterocigosidad esperada de 0.354 en una colección de líneas de Capsicum annuum analizadas con microsatélites, lo que evidencia cierta variación genética aun en líneas homocigóticas.

Contrario a lo esperado, la heterocigosidad fue más baja en el híbrido (Cuadro 3), lo cual podría deberse al parentesco entre progenitores y al nivel de homocigosis de sus líneas. Los datos anteriores indican que los tipos nativos, principalmente 'Poblano, 'Ancho' y 'Miahuateco', poseen la mayor diversidad genética y por ende un mayor potencial para utilizarse en programas de conservación o mejoramiento.

\section{Diferenciación genética entre variedades y tipos}

El tipo 'Miahuateco' y el híbrido presentaron los valores de $\mathrm{F}_{\mathrm{IS}}$ más bajos ( -0.202 y -0.723 respectivamente), que indican que poseen un mayor número de individuos heterocigotos dentro de cada población, bajo el supuesto de equilibrio de 
Cuadro 2. Loci de microsatélites e iniciadores utilizados para su amplificación por PCR en muestras de chile (Capsicum annuum L.).

\begin{tabular}{|c|c|c|c|c|}
\hline Grupo & Locus & Unidad repetitiva & Iniciadores (Delante/Reversa) & Tamaño $(\mathrm{pb})$ \\
\hline \multirow{4}{*}{1} & Hpms1-106 & $\left(\right.$ AAAAAT $_{4}$ & $\begin{array}{l}\text { HEX-tccaaactacaagcctgcctaacc/ } \\
\text { ttttgcattattgagtcccacagc }\end{array}$ & $158-164$ \\
\hline & Hpms2-2 & $(\mathrm{GT})_{9}$ & $\begin{array}{l}\text { 6-FAM-gcaaggatgcttagttgggtgtc/ } \\
\text { tcccaaaattaccttgcagcac }\end{array}$ & $148-151$ \\
\hline & Hpms2-24 & $(\mathrm{CT})_{17}(\mathrm{CA})_{5} \mathrm{~A}_{21}$ & $\begin{array}{l}\text { 6-FAM-tcgtattggcttgtgatttaccg/ } \\
\text { ttgaatcgaatacccgcaggag }\end{array}$ & $187-205$ \\
\hline & HpmsCaSIG19 & $\begin{array}{l}(\mathrm{CT})_{6}(\mathrm{AT})_{8} \\
(\mathrm{GTAT})_{5}\end{array}$ & $\begin{array}{l}\text { HEX-catgaatttcgtcttgaaggtccc/ } \\
\text { aagggtgtatcgtacgcagcctta }\end{array}$ & $216-223$ \\
\hline \multirow[b]{2}{*}{2} & Hpms1-5 & $(\mathrm{AT})_{11}(\mathrm{GT})_{17}$ & $\begin{array}{l}\text { 6-FAM-ccaaacgaaccgatgaacactc/ } \\
\text { gacaatgttgaaaaaggtggaagac }\end{array}$ & $289-323$ \\
\hline & Hpms1-62 & $(\mathrm{TG})_{23}(\mathrm{AG})_{9}$ & $\begin{array}{l}\text { 6-FAM-catgaggtctcgcatgatttcac/ } \\
\text { ggagaaggaccatgtactgcagag }\end{array}$ & 184-192 \\
\hline \multirow{2}{*}{3} & Hpms1-173 & $(\mathrm{GA})_{16}(\mathrm{TG})_{2}$ & $\begin{array}{l}\text { HEX-tgctgggaaagatctcaaaagg/ } \\
\text { atcaaggaagcaaaccaatgc }\end{array}$ & $159-169$ \\
\hline & AF244121 & $\begin{array}{l}(\mathrm{TTG})_{5 \mathrm{IMP}}{ }^{\dagger} \\
(\mathrm{AT})_{6 \mathrm{IMP}}(\mathrm{GT})_{3 \mathrm{IIMP}}\end{array}$ & $\begin{array}{l}\text { 6-FAM-tacctcctcgccaatccttctg/ } \\
\text { ttgaaagttctttcatgacaacc }\end{array}$ & $240-243$ \\
\hline \multirow{2}{*}{4} & Hpms1-1 & $(\mathrm{CA})_{12}(\mathrm{TA})_{4}$ & $\begin{array}{l}\text { 6-FAM-tcaacccaatattaaggtcacttcc/ } \\
\text { ccaggcggggattgtagatg }\end{array}$ & $270-284$ \\
\hline & Hpms1-274 & $(\mathrm{GTT})_{7}$ & $\begin{array}{l}\text { HEX-tcccagacccetcgtgatag/ } \\
\text { tcctgctccttccacaactg }\end{array}$ & $162-180$ \\
\hline \multirow{9}{*}{5} & Hpms1-172 & $(\mathrm{GA})_{15}$ & $\begin{array}{l}\text { HEX-gggtttgcatgatctaagcatttt/ } \\
\text { cgctggaatgcattgtcaaaga }\end{array}$ & $335-346$ \\
\hline & CM0005 & $(\mathrm{CCA})_{8}$ & $\begin{array}{l}\text { 6-FAM-catgaccaccatgaggata / } \\
\text { gatagccacgagcatagtatt }\end{array}$ & $154-160$ \\
\hline & Hpms1-143 & $(\mathrm{AG})_{12}$ & $\begin{array}{l}\text { 6-FAM-aatgctgagctggcaaggaaag/ } \\
\text { tgaaggcagtaggtggggagtg }\end{array}$ & $220-232$ \\
\hline & Hpms1-148 & $(\mathrm{GA})_{14}$ & $\begin{array}{l}\text { HEX-ggcggagaagaactagacgattagc/ } \\
\text { ccacccaatccacatagacg }\end{array}$ & 184-195 \\
\hline & Hpms1-214 & $\begin{array}{l}(\text { GTTT) } \\
(\text { TTG })_{9}\end{array}$ & $\begin{array}{l}\text { 6-FAM-tgcgagtaccgagttctttctag/ } \\
\text { ggcagtcctgggacaactcg }\end{array}$ & $88-108$ \\
\hline & Hpms2-21 & $\begin{array}{l}(\mathrm{AT})_{11}(\mathrm{AC})_{9} \\
(\mathrm{ATAC})_{10}\end{array}$ & $\begin{array}{l}\text { HEX-tttttcaattgatgcatgaccgata/ } \\
\text { catgtcattttgtcattgatttgg }\end{array}$ & $264-295$ \\
\hline & HpmsAT2-14 & $(\mathrm{AAT})_{16 \mathrm{IMP}}$ & $\begin{array}{l}\text { 6-FAM-tttagggtttccaactcttcttcc/ } \\
\text { ctaaccccaccaagcaaaacac }\end{array}$ & 176 \\
\hline & AF039662 & $\mathrm{T}_{26 \mathrm{MMP}}$ & $\begin{array}{l}\text { 6-FAM-cccctcgtctctctttattt/ } \\
\text { ttgcaaatcttttgtcaatttt }\end{array}$ & $104-116$ \\
\hline & CAN130829 & $\mathrm{T}_{16}$ & $\begin{array}{l}\text { 6-FAM-gctaattacttgctccgttttg/ } \\
\text { aatgggggagtttgttttgg }\end{array}$ & $182-185$ \\
\hline
\end{tabular}

${ }^{\dagger}$ Repetición imperfecta. 
Cuadro 3. Número de alelos por locus, proporción de loci polimórficos y heterocigosidad esperada, estimados a partir de los 19 loci de microsatélites evaluados.

\begin{tabular}{|c|c|c|c|c|c|c|c|}
\hline Tipo & $\begin{array}{c}\text { Núm. de } \\
\text { poblaciones }\end{array}$ & $\begin{array}{l}\text { Núm. de } \\
\text { alelos }\end{array}$ & $\begin{array}{l}\text { Alelos por } \\
\text { locus }\end{array}$ & Desv. est. & $\begin{array}{c}\% \text { de loci } \\
\text { polimorficos }\end{array}$ & $\begin{array}{l}\text { Heterocigosidad } \\
\text { esperada }\end{array}$ & Desv. est. \\
\hline 'Poblano' & 55 & 100 & 5.26 & 3.14 & 94.74 & 0.442 & 0.267 \\
\hline 'Ancho' & 3 & 67 & 3.53 & 1.74 & 94.74 & 0.469 & 0.215 \\
\hline 'Loco' & 2 & 56 & 2.95 & 1.87 & 73.68 & 0.380 & 0.280 \\
\hline 'Miahuateco' & 2 & 56 & 2.95 & 1.31 & 94.74 & 0.477 & 0.168 \\
\hline Híbrido & 1 & 29 & 1.61 & 0.85 & 42.11 & 0.207 & 0.266 \\
\hline Total & 63 & 105 & 5.53 & 3.10 & 80.00 & 0.395 & 0.180 \\
\hline
\end{tabular}

Hardy-Weinberg. Estas variedades también presentaron el valor de $\mathrm{F}_{\mathrm{IT}}$ más bajo, lo que sugiriere una menor pérdida de heterocigotos; el híbrido mostró valores notablemente más bajos de $\mathrm{F}_{\mathrm{IT}}$, debido a la naturaleza de su origen. Por el contrario, el tipo 'Poblano' presentó los valores más altos de $\mathrm{F}_{\mathrm{IS}} \mathrm{y} \mathrm{F}_{\mathrm{IT}}$, lo que revela que posee mayor número de homocigotos y mayor pérdida de heterocigosidad.

Los tipos 'Miahuateco' y 'Poblano' presentaron una diferenciación genética $\left(\mathrm{F}_{\mathrm{ST}}\right)$ moderada, de 0.065 y 0.124 , respectivamente, mientras que esta diferenciación en los tipos 'Ancho' y 'Loco' fue relativamente alta, de 0.176 y 0.156. Estos resultados indican que existe una alta diferenciación entre las poblaciones de cada variedad y por ende un reducido flujo génico entre ellas (Snyder et al., 1985). Con un valor general de $\mathrm{F}_{\mathrm{ST}}$ de 0.108 para todos los grupos, se infiere que 89.2 \% de la variación total se encuentra dentro de las poblaciones, y sólo $10.8 \%$ entre ellas (Cuadro 4). Estos valores permiten inferir que un programa de selección recurrente intrapoblacional podría ser adecuado para el aprovechamiento de la diversidad existente con fines de mejoramiento, que realizado a nivel de microrregiones implicaría la utilización de un elevado número de poblaciones nativas sobresalientes, con la consecuente conservación de la diversidad.

Cuadro 4. Estadísticos de $\mathrm{F}$ calculados a partir de los 19 loci de microsatélites para los cinco tipos de chile (Capsicum annumm L.).

\begin{tabular}{lccc}
\hline Tipo & $\mathrm{F}_{\mathrm{IS}}{ }^{\dagger}$ & $\mathrm{F}_{\mathrm{IT}}{ }^{\dagger \dagger}$ & $\mathrm{F}_{\mathrm{ST}}{ }^{\prime}$ \\
\hline 'Poblano' & 0.205 & 0.316 & 0.140 \\
'Ancho' & -0.055 & 0.131 & 0.176 \\
'Loco' & 0.029 & 0.182 & 0.158 \\
'Miahuateco' & -0.202 & -0.124 & 0.065 \\
Híbrido & -0.723 & -0.723 & 0.000 \\
General & -0.149 & -0.044 & 0.108 \\
\hline
\end{tabular}

${ }^{\dagger}$ Desviación de heterocigosidad dentro de accesiones; ${ }^{\dagger+}$ Diferenciación de accesiones; ${ }^{9}$ Coeficiente global de endogamia.

Resultados parecidos en cuanto a la distribución de la diversidad, fueron reportados por Oyama et al. (2006) en poblaciones domesticadas de chile 'Serrano,' 'Jalapeño' y 'Morrón' del noroeste mexicano analizadas con RAPDs, ya que estas poblaciones mostraron diferenciación genética
$\left(\mathrm{G}_{\mathrm{ST}}\right)$ de 0.32 , que indica que $67.8 \%$ de la variabilidad se encuentra dentro de las poblaciones, y $32.2 \%$ entre ellas. La mayor variabilidad genética se encontro dentro de las poblaciones domesticadas estudiadas por Oyama et al. (2006) y también reportada por Hernández et al. (2006), quienes con isoenzimas encontraron una diferenciación genética $\left(G_{\mathrm{ST}}\right)$ de 0.17 en las poblaciones.

\section{Relaciones entre variedades}

Con base en el análisis de componentes principales (CP), los primeros 10 componentes principales explicaron 50.5 $\%$ de la variación total (Cuadro 5). El primer componente principal (CP1) explicó $14 \%$ de dicha variación, mientras que CP2 y CP3 explicaron 5.5 y $5.1 \%$, respectivamente. En contraste, con caracterización morfológica el mayor porcentaje de la variación observada es típicamente explicada por los tres primeros componentes principales. Al respecto, Castañón-Nájera et al. (2008) encontraron que los tres primeros componentes principales explicaron $78.5 \%$ de la varianza total en chile, y Moreno et al. (2007) indicaron que $57.5 \%$ de la variación observada fue explicado por los tres primeros componentes principales en chile 'Guajillo'.

Cuadro 5. Valores propios y proporción de varianza explicada por los primeros 10 componentes principales generados a partir de la matriz de correlación de los 105 alelos detectados para 19 loci de microsatélites en 63 poblaciones de chile (Capsicum annumm L.).

\begin{tabular}{cccc}
\hline CP & Valores propios & Proporción de varianza & Varianza acumulada \\
\hline 1 & 14.712 & 0.142 & 0.142 \\
2 & 5.955 & 0.057 & 0.199 \\
3 & 5.285 & 0.051 & 0.250 \\
4 & 4.723 & 0.045 & 0.295 \\
5 & 4.185 & 0.040 & 0.335 \\
6 & 4.058 & 0.039 & 0.374 \\
7 & 3.726 & 0.036 & 0.410 \\
8 & 3.490 & 0.034 & 0.444 \\
9 & 3.405 & 0.033 & 0.476 \\
10 & 3.021 & 0.029 & 0.505 \\
\hline
\end{tabular}

Los loci Hpms2-2, Hpms1-214 y AF244121 fueron los más importantes, ya que todos o la mayoría de sus alelos contribuyeron en los tres primeros componentes principales 
para la definición de la variabilidad. La dispersión de las poblaciones dibujada en un plano cartesiano definido por $\mathrm{CP} 1$ y $\mathrm{CP} 2$, permitió mostrar que las poblaciones pertenecientes al tipo 'Poblano' tienden a agruparse para formar un conglomerado medianamente compacto entre el segundo y tercer cuadrante (Figura 1). Algunas poblaciones del tipo 'Poblano' no quedaron incluidas en ninguno de los grupos, como la población FT3-P (cuadrante IV, izquierda) tipo 'Poblano', por lo que es probable que comparta algunas características del tipo 'Loco'; la población LG5-P (cuadrante II), que corresponde a un chile conocido localmente como 'Poblano Negro' y la población CH1-P (cuadrante IV, derecha), fueron las más diferenciadas entre las poblaciones del tipo 'Poblano', por los alelos que más influenciaron al primer componente principal.

En el mismo contexto, se aprecia que la población MC8-A del tipo 'Ancho', fue incluida en el grupo mayoritario del tipo 'Poblano', en tanto que las poblaciones RG1-A y RG2-A del tipo 'Ancho', provenientes del Estado de Zacatecas, el híbrido comercial 'Doroteo', las poblaciones MI1-M y MI2-M del tipo 'Miahuateco', procedentes de Tehuacán, Puebla, así como las poblaciones del tipo 'Loco' FT4-L y MC7-L, estuvieron delimitadas claramente (Figura 1).

La extensa distribución dentro del tipo 'Poblano' sugiere que no hay una clara diferenciación dentro de las poblaciones, lo que fue corroborado por un bajo valor del estadístico $\mathrm{F}_{\mathrm{ST}}(0.140)$ para este grupo de chiles (Cuadro 4), comportamiento diferente entre los tipos de chile que mostraron delimitaciones definidas. Este resultado es atribuible a los diferentes esquemas de selección aplicados por parte de los agricultores (Oyama et al., 2006) durante el proceso de domesticación.

Los agrupamientos anteriores fueron similares a los derivados del análisis de conglomerados (Figura 2), lo que indica una alta consistencia en la definición de las asociaciones. A una distancia genética de 0.28 se distinguen dos poblaciones aisladas y tres grupos de chile. El primer

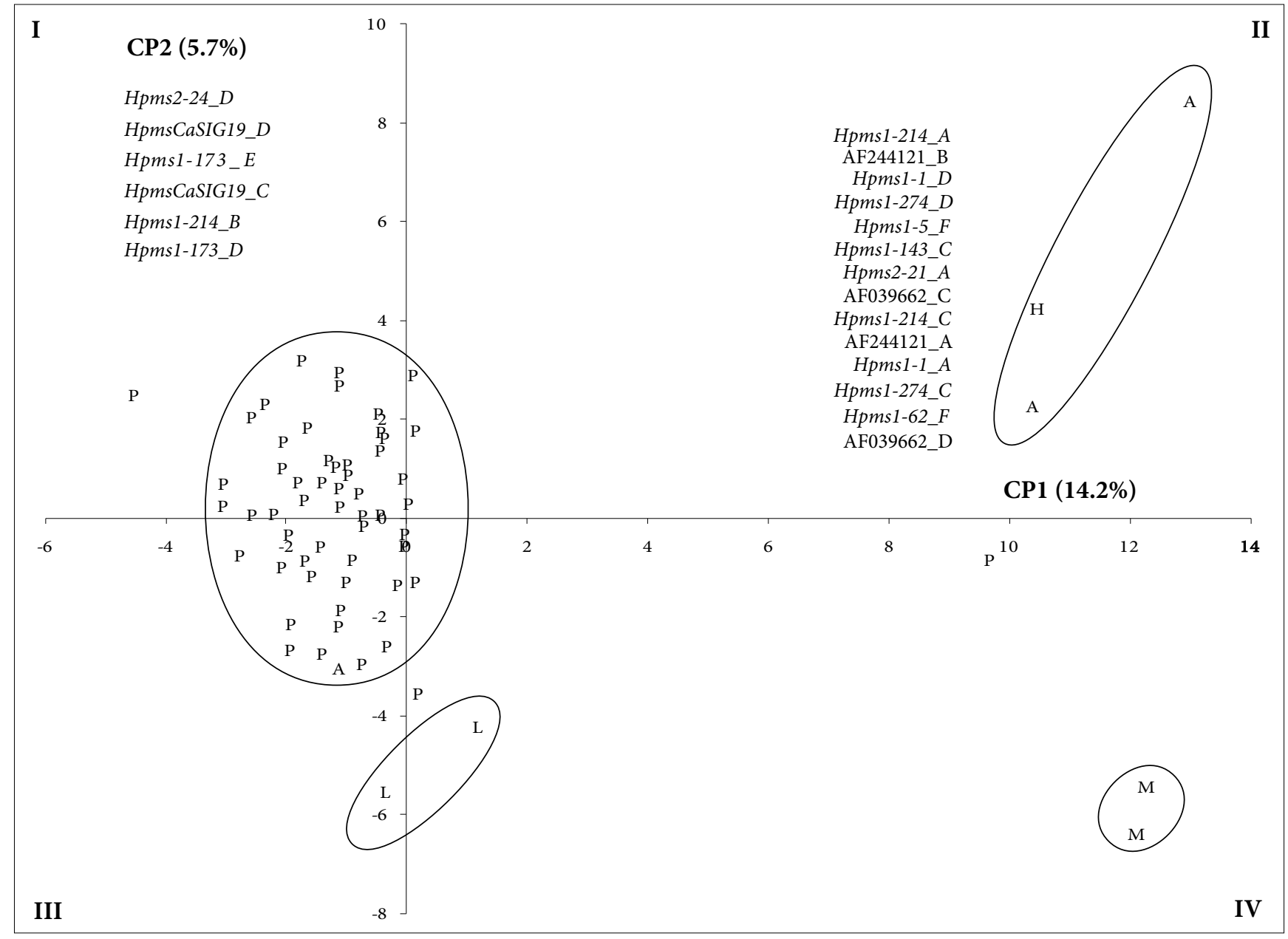

Figura 1. Dispersión de 63 poblaciones de chile con base en los dos primeros componentes principales derivados de 105 alelos encontrados en 19 loci de SSRs. Se indican los alelos de mayor influencia en cada componente principal. 


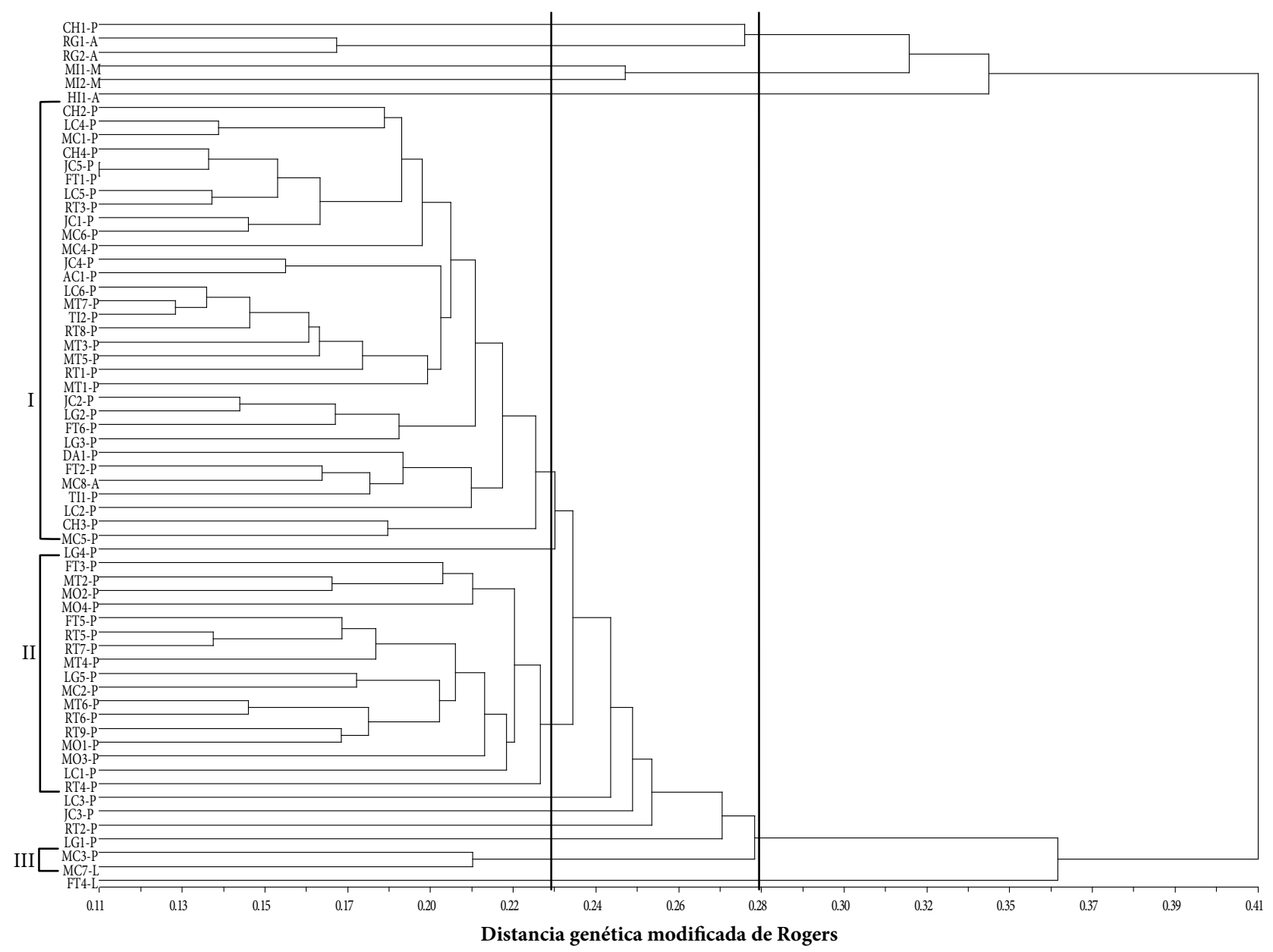

Figura 2. Dendrograma de las 63 poblaciones de chile (Capsicum annuum L.) basado en 105 alelos de SSRs mediante el uso de la distancia modificada de Rogers y el método de agrupamiento UPGMA.

grupo se conformó por las poblaciones RG1-A y RG2-A del tipo 'Ancho' de Zacatecas, y la población de tipo 'Poblano' $\mathrm{CH} 1-\mathrm{P}$, aunque ésta última se encuentra notablemente menos relacionada. En el segundo grupo se asociaron las poblaciones de tipo 'Miahuateco' MI1-M y MI2-M provenientes de Tehuacán, Puebla. El híbrido comercial HI1-A se ubicó de forma independiente, al igual que la población FT4-L del tipo 'Loco' (Figura 2).

El tercer grupo se conformó por el resto de las poblaciones locales, entre las cuales pueden distinguirse tres subgrupos a una distancia genética de 0.23 . El subgrupo I incluyó a la gran mayoría de las poblaciones procedentes de las principales localidades productoras de chile 'Poblano', como son Cháhuac, Domingo Arenas, Juárez Coronaco, San Antonio Chautla, San Lorenzo Chiautzingo, San Lucas el Grande, San Mateo Capultitlán y San Miguel Tianguistengo, con excepción de las poblaciones LC1-P, LG5-P y MC2-P que se ubicaron en el subgrupo II, y las poblaciones JC3-P, LC3-P, LG1-P y LG4-P ('Poblano Rojo') que se ubicaron aisladas.
En el subgrupo II se encontraron la mayoría de las poblaciones originarias de San Rafael Tlanalapan y Santa María Moyotzingo, ambas en el municipio de San Martín Texmelucan, con excepción de las poblaciones RT1-P, RT3-P y RT8-P que se asociaron en el subgrupo I, y la población RT2-P que no se integró. Las poblaciones de San Felipe Teotlatzingo y San Matías Tlalancaleca se distribuyeron dispersas entre ambos subgrupos. No fue posible obtener un patrón definido en la distribución de las poblaciones dentro de estos dos subgrupos.

El subgrupo III se conformó con las poblaciones MC3-P y MC7-L (Figura 2), esta última clasificada como del tipo 'Loco' e incluida junto con la población FT4-L del mismo tipo, en el análisis de componentes principales. Es factible entonces que la primera también posea características de chile 'Loco'. Según Oyama et al. (2006), a una distancia genética de 0.254 se pueden separar diferentes poblaciones domesticadas de los chiles 'Serrano', 'Jalapeño' y 'Morrón', lo que indica que se encuentran claramente diferenciadas. Resultados similares fueron obtenidos por 
Hernández et al. (2006) en las mismas poblaciones, al estar diferenciadas por una distancia de 0.212 .

\section{CONCLUSIONES}

En Puebla, como en la mayoría de las regiones de cultivo de los chiles en México, las variedades cultivadas de chile 'Poblano' se comportan como poblaciones consanguíneas. El chile 'Poblano' es de los escasos tipos de chile cuya concentración de capsaicinoides no es su principal interés de cultivo. Los 19 loci de microsatélites evaluados en las poblaciones permitieron diferenciar las variedades provenientes de la Sierra Nevada de Puebla, Tehuacán, Puebla y Rancho Grande, Zacatecas, y aparte el híbrido comercial. Las variedades locales exhibieron complejidad genética mostrada por una mayor dispersión a través del plano cartesiano de componentes principales, las cuales no mostraron un patrón definido de distribución, y por ello hubo una mayor variabilidad en los chiles del tipo 'Poblano'. Se determinó que $89.2 \%$ de la variación total está dentro de las poblaciones y sólo $10.8 \%$ entre ellas, lo cual es acorde con su sistema de apareamiento o biología floral. Estos valores son indicativos de que un programa de selección recurrente dentro de poblaciones sería adecuado para un aprovechamiento más eficiente de la diversidad existente.

\section{BIBLIOGRAFÍA}

Applied Bisystems (2005) GeneMapper® Software Version 4.0. Reference and Troubleshooting Guide. Applied Biosystems Inc. Foster City, CA. 82 p.

Castañón-Nájera G, L Latournerie-Moreno, M Mendoza-Elos, A VargasLópez, H Cárdenas-Morales (2008) Colección y caracterización de chile (Capsicum spp.) en Tabasco, México. Phyton 77:189-202.

Djian-Caporalino, V Lefebvre, A M Sage-Daubèze, A Palloix (2006) Capsicum. In: Genetic Resources, Chromosome Engineering and Crop Improvement. Vol. 3. Vegetable Crops. R J Singh (ed.). CRC Press. Boca Raton, FL. pp:185-243.

FAOSTAT, FAO Statistical Database (2009) FAO Statistical Databases. Publishing Management Service, Information Division, Food and Agricultural Organization of the United Nations (FAO). Via delle Terme di Caracalla, Rome, Italy. http://faostat.fao.org. ( julio de 2011).
Hanáček P, T Vyhnánek, M Rohrer, J Cieslarová, H Stavělíková (2009) DNA polymorphism in genetic resources of red pepper using microsatellite markers. Hort. Sci. (Prague) 36:127-132.

Hernández V S, A González R, P Sánchez P, A Casas, K Oyama (2006) Estructura y diferenciación genética de poblaciones silvestres y domesticadas de chile del noroeste de México analizada con isoenzimas y RAPDs. Rev. Fitotec. Mex. 29 (Núm. Esp. 2):25-29.

Kwon Y S, J M Lee, G B Yi, S I Yi, K M Kim, E H Soh, K M Bae, E K Park, I H Song, B D Kim (2005) Use of SSR markers to complement tests of distinctiveness, uniformity, and stability (DUS) of pepper (Capsicum annuum L.) varieties. Molec. Cells 19:428-435.

Lee J M, S H Nahm, Y M Kim, B D Kim (2004) Characterization and molecular genetic mapping of microsatellite loci in pepper. Theor. Appl. Genet. 108: 619-627.

Moreno P E C, O Cruz Á, C H Avendaño A, M A T Martínez D, A Peña L (2007) Morphological variation in Guajillo chili pepper plants (Capsicum annuum L.). African Crop Sci. Conf. Proc. 8:327-332.

Oyama K, S Hernández-Verdugo, C Sánchez, A González-Rodríguez, P Sánchez-Peña, J A Garzón-Tiznado, A Casas (2006) Genetic structure of wild and domesticated populations of Capsicum annuum (Solanaceae) from northwestern Mexico analyzed by RAPDs. Genet. Resour. Crop Evol. 53:553-562.

Pickersgill B (1991) Cytogenetics and evolution of Capsicum L. In: Chromosome Engineering in Plants, Genetics, Breeding and Evolution. Part B. T Tsuchiya, P K Gupta (eds). Elsevier. Amsterdam. pp:139-160.

Rodríguez J, B V Peña O, A Gil M, B Martínez C, F Manzo, L Salazar L (2007) Rescate in situ del chile 'Poblano' en Puebla, México. Rev. Fitot. Mex. 30:25-32.

Rohlf F J (2002) NTSYS pc: Numerical Taxonomy System, Version 2.1. Exeter Publishing, Setauket, NY.

SAS Institute (2002) SAS/STAT User's Guide, Software version 9.0. Cary, N.C. USA. $4424 \mathrm{p}$.

SDR Puebla, Secretaría de Desarrollo Rural Puebla (2007) Cadenas Productivas Agropecuarias y Acuícolas del Estado de Puebla. Primera Edición. Gobierno del Estado de Puebla. México. 97 p.

Snyder L A, D Freifelder, D L Hartl (1985) General Genetics. Jones \& Bartlett. Boston, MA. 666 p.

Tam S M, C Mhiri, A Vogelaar, M Kerkveld, S R Pearce, M A Grandbastien (2005) Comparative analyses of genetic diversity within tomato and pepper collections detected by retrotransposon-based SSAP, AFLP, and SSR. Theor. Appl. Genet. 110:819-831.

Wright S (1978) Evolution and the Genetics of Populations. Vol. 4. Variability Within and Among Natural Populations. University of Chicago Press. Chicago, IL. 510 p.

Yeh F C, R Yang, T Boyle (1999) POPGENE Version 1.31. Microsoft Window-based Freeware for Population Genetic Analysis. Quick User Guide. University of Alberta and Centre for International Forestry Research. Edmonton, Canada. Disponible en: http:// www.ualberta.ca/ fyeh/popgne.pdf. (Enero 2011). 